\title{
Soft-x-ray resonant scattering from V/Fe (001) magnetic superlattices
}

\author{
Maurizio Sacchi and Alessandro Mirone \\ LURE, Centre Universitaire Paris-Sud, Bâtiment 209D, Boîte Postale 34, F-91898 Orsay Cedex, France \\ Coryn F. Hague* and Jean-Michel Mariot \\ Laboratoire de Chimie, Physique-Matière et Rayonnement (UMR 7614), Université Pierre et Marie Curie, 11 rue Pierre et Marie Curie, \\ F-75231 Paris Cedex 05, France \\ Luca Pasquali \\ Dipartimento di Fisica dell'Università and INFM, Via Campi 213/a, I-41100 Modena, Italy \\ Peter Isberg ${ }^{\dagger}$ \\ Department of Physics, Uppsala University, Box 530, S-751 21, Uppsala, Sweden \\ Eric M. Gullikson and James H. Underwood \\ Center for X-Ray Optics, Lawrence Berkeley National Laboratory, University of California, Berkeley, California 94720 \\ (Received 19 April 1999)
}

\begin{abstract}
The magnetic properties of $\mathrm{Fe}$ and $\mathrm{V}$ in ( $n \mathrm{ML} \mathrm{V} \mathrm{/} 5 \mathrm{ML} \mathrm{Fe}$ ) (001) superlattices ( $n=1,2$, and $5 \mathrm{ML}$ ) have been investigated by measuring the magnetization dependent scattering of elliptically polarized soft $\mathrm{x}$ rays at various scattering angles and for photon energies across the $2 p$ x-ray-absorption edges of both Fe and V. A quantitative estimate of the magnetic moment induced on vanadium is obtained from the analysis of the resonant scattering data. [S0163-1829(99)50842-4]
\end{abstract}

The magnetic properties of artificially structured devices (thin layers, superlattices, or arrays of microdots) can be tailored to specific needs using appropriate elements, growth modes, or periodic structures. Among them, metallic multilayers, featuring oscillating magnetic coupling and giant magnetoresistance effects, are preeminent. ${ }^{1}$ Not only are these systems currently under study by theorists and experimentalists, ${ }^{2,3}$ but they are also under development for new magnetic recording devices. ${ }^{4}$

$\mathrm{V} / \mathrm{Fe}$ superlattices are a good example of how a multilayer device can present a whole variety of magnetic behavior, depending on the layer thicknesses $t_{\mathrm{V}}$ and $t_{\mathrm{Fe}}$, and on the growth orientation. Concerning the (001) plane, ferromagnetic coupling between successive Fe layers has been reported for $t_{\mathrm{V}}$ up to about 12 monolayers (ML), ${ }^{5}$ while antiferromagnetic coupling has been observed for thin Fe layers (about $3 \mathrm{ML}$ ) when the vanadium spacers exceed 12 ML. ${ }^{6}$ In both cases, the samples have two in-plane easy axes along the [100] and [010] directions. When grown in the (110) plane, the V/Fe system exhibits uniaxial in-plane anisotropy and a magnetic coupling between Fe layers that still depends on the $\mathrm{V}$ thickness. 7

Until recently, the magnetic properties of $\mathrm{V}$ in these layers have been the subject of some controversy due to the fact that it is difficult to investigate the weak magnetism of $\mathrm{V}$ in a superstructure containing a large amount of Fe. Yet, it is important to understand the magnetic coupling between $\mathrm{V}$ and $\mathrm{Fe}$, and to quantify the magnetic moment carried by $\mathrm{V}$ in real structures. Using electron spin-polarization analysis, $\mathrm{V}$ has been shown to acquire a magnetic moment when in contact with Fe, e.g., when deposited on an Fe crystal. ${ }^{8}$ When it comes to probing buried interfaces, such techniques are no longer operational. Up to now, the only studies of $\mathrm{V}$ magnetism in multilayers have been performed by circular dichroism at the $2 p$ absorption edge, ${ }^{9,10}$ detecting the sample drain current or, equivalently, the total electron yield (TEY). Certainly circular dichroism in X-ray-absorption spectroscopy (XAS) is a powerful technique because of its element selectivity and the access it provides to a quantitative evaluation of magnetic moments via the application of sum rules, ${ }^{11,12}$ but the TEY detection mode has several drawbacks that have been discussed in the past. ${ }^{13}$

In this paper, we propose a different experimental approach, based on X-ray resonant magnetic scattering (XRMS), which takes full advantage of all aspects of dichroism in X-ray absorption, along with some additional technical and conceptual points of interest. As in XAS, photons are tuned to core excitation resonances, providing element selectivity, and $3 d$ magnetic orbitals are probed directly by the $2 p \rightarrow 3 d$ dipole transitions. In addition, soft-x-ray wavelengths match the typical spacings of metallic multilayers, so Bragg diffraction experiments under resonant conditions may be performed. ${ }^{14,15}$ Moreover, XRMS may be used to distinguish between magnetic arrangements with different magnetization profiles, even when they have the same average properties. ${ }^{16}$ Another relevant aspect of XRMS, when dealing with magnetic multilayers, is that it is a photon-inphoton-out experiment which means that the presence of applied magnetic fields does not affect the measurements. Moreover, the probing depth is only limited by the photon path within the sample and no saturation effects need to be accounted for. The large probing depth is very important if we wish to extract bulk properties from capped multilayer samples (i.e., not those just related to the topmost layers). 
TABLE I. Structural [2 $2 d$ spacing, thickness ratio $\gamma=t_{\mathrm{Fe}} /\left(t_{\mathrm{Fe}}\right.$ $\left.+t_{\mathrm{V}}\right)$, and thickness $\left.t\right]$ and magnetic properties of $\left(\mathrm{V}_{n} / \mathrm{Fe}_{5}\right)_{m}$ superlattices.

\begin{tabular}{cccccccc}
\hline \hline$n$ & $m$ & $2 d(\AA)$ & $\gamma$ & $t(\AA)$ & $\left\langle l_{z}\right\rangle_{\mathrm{V}}$ & $\left\langle s_{z}\right\rangle_{\mathrm{V}}$ & $\left\langle\mu_{z}\right\rangle_{\mathrm{V}}$ \\
\hline 1 & 80 & 16.5 & 0.83 & 660 & -0.08 & 0.37 & 0.66 \\
2 & 60 & 22.2 & 0.71 & 666 & -0.05 & 0.22 & 0.40 \\
5 & 40 & 30.6 & 0.50 & 612 & -0.03 & 0.15 & 0.26 \\
\hline \hline
\end{tabular}

We report on XRMS spectra collected for several scattering angles $\theta$ from $\mathrm{V} / \mathrm{Fe}$ superlattices of the type ( $n \mathrm{ML} \mathrm{V} \mathrm{/}$ $5 \mathrm{ML} \mathrm{Fe}$ ) (denoted hereafter as $\mathrm{V}_{n} / \mathrm{Fe}_{5}$ ). Whenever possible we have included $\theta$ values satisfying Bragg conditions at resonant energies. Data were analyzed using the calculational scheme described previously. ${ }^{17}$ Within the limitations discussed in the text, we have also applied sum rules ${ }^{11,12}$ in order to obtain a quantitative estimate of the magnetic moment induced on $\mathrm{V}$.

The superlattices were epitaxially grown by sputter deposition of the metallic layers on $\mathrm{MgO}$ (001) single-crystal substrates. Their final structure is $\left(\mathrm{V}_{n} / \mathrm{Fe}_{5}\right)_{m}$ with $(n=1, m$ $=80),(n=2, m=60)$, and $(n=5, m=40)$. Their structural properties have been described previously, ${ }^{6,18}$ and the relevant parameters are reported in Table I. The easy axes of magnetization are always along the [110] axes of the substrate.

XRMS measurements were performed at the Fe and V $2 p$ edges on beamline 6.3.2 at ALS (Berkeley), ${ }^{19}$ using elliptically polarized light $(40 \%$ circular polarization rate and a resolving power of $\approx 2000$ ). The external magnetic field $(\approx 0.1 \mathrm{~T}$, applied along an easy axis, in the sample surface and in the scattering plane) was reversed after each complete scan of the chosen spectral region. More details about the experimental apparatus can be found in previous papers. ${ }^{15}$

We also measured the absorption spectra and circular dichroism under the same experimental conditions. The drain current detection method has a very short probing depth [about $15 \AA$ in Fe (see Ref. 13)]. This implies that we are only measuring a few layers of our sample. Moreover, only the outermost layers are probed which are more likely to be affected by inhomogeneities, contamination, or by the presence of the capping layer ( $\mathrm{Pd}$, in our case). We have used the measured absorption line shapes to build up the dielectric tensor for Fe and V that will be employed in the analysis of XRMS data, leaving as a free parameter a multiplication factor $\left(R_{\mathrm{V}}, R_{\mathrm{Fe}}\right)$ on the magnetization dependent part of the optical constants. Thanks to the linearity of Kramers-Kronig transformations, this parameter is the same for the real (dispersive) and imaginary (absorptive) parts of the refractive index. Using the computational method described in a previous paper, ${ }^{17}$ we fit, for each sample, $R_{\mathrm{Fe}}$ and $R_{\mathrm{V}}$ to the ensemble of the energy- and angle-dependent scattering curves, including resonant Bragg diffraction spectra.

All resonant spectra showed magnetization dependence. Figure 1, for instance, shows the energy-dependent reflectivity curves for the $\mathrm{V}_{1} / \mathrm{Fe}_{5}$ sample: the two panels refer to the $\mathrm{V}$ and Fe $2 p$ edges, measured at $\theta=10^{\circ}$. Full and dashed lines are the sum and the difference of reflectivity curves obtained for opposite magnetizations. The difference curves for $\mathrm{V}$ and $\mathrm{Fe}$ have similar line shapes but opposite signs,

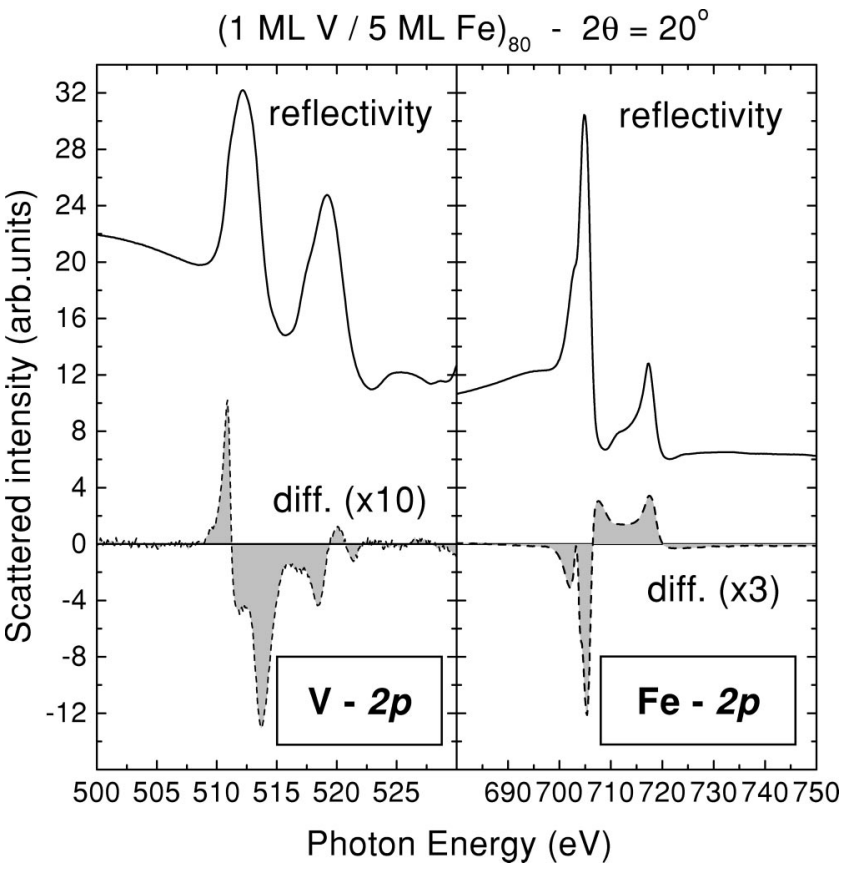

FIG. 1. Reflectivity at the $\mathrm{V}$ and $\mathrm{Fe} 2 p$ resonances for the $\mathrm{V}_{1} / \mathrm{Fe}_{5}$ superlattice at $2 \theta=20^{\circ}$ (full lines: magnetization averaged curves; dotted lines: difference curves).

already suggesting an antiparallel alignment between $\mathrm{Fe}$ and $\mathrm{V}$ magnetic moments. Such a conclusion, though, can be reached safely only after a thorough analysis of the complete set of data, including angular dependence. Note that the fine structure that can be observed at the Fe edges is directly related to the morphology of the sample and depends on the scattering angle.

$R_{\mathrm{Fe}}$ values, obtained by fitting XRMS spectra at the $\mathrm{Fe} 2 p$ edge, were such that the scaled dichroism curves in absorption did not deviate appreciably from that of a bulk Fe reference. Applying sum rules, we obtained values of between $2.1 \mu_{B}$ and $2.3 \mu_{B}$ for the magnetic moment per Fe atom, depending on choices made in the data reduction (see below). We conclude that for these $\mathrm{V}_{n} / \mathrm{Fe}_{5}$ multilayers there is no measurable change in the $\mathrm{Fe}$ average magnetic moment as a function of $\mathrm{V}$ thickness. This also implies a ferromagnetic alignment of all the Fe layers in the superstructure.

The magnetic moment carried by $\mathrm{V}$, if any, is small in a sample containing ferromagnetic iron, hence element selectivity is at a premium. We were able to extract $R_{\mathrm{V}}$ to a high degree of confidence by means of a fitting procedure over the entire set of energy-dependent reflectivity curves taken at several scattering angles. Figure 2 compares experimental and calculated asymmetry ratio curves at a few scattering angles for the $\mathrm{V}_{1} / \mathrm{Fe}_{5}$ sample. Since we only optimize a scaling factor on the dichroism curve, discrepancies persist between calculation and experiment, but the general agreement over the ensemble of resonant scattering data is good. The expected asymmetry ratios at the V $2 p$ edges for $n=2$ and $n=5$ (dashed lines) are compared to experiment (squares) in Fig. 3. No further parametrization has been introduced. This confrontation indicates that if we assume the same magnetic moment as for $n=1$, dichroism for $n=2$ and $n=5$ is overestimated. Best fits are obtained by reducing $R_{\mathrm{V}}$ to $60 \%$ and $40 \%$ of its initial value, respectively. Since, for a 


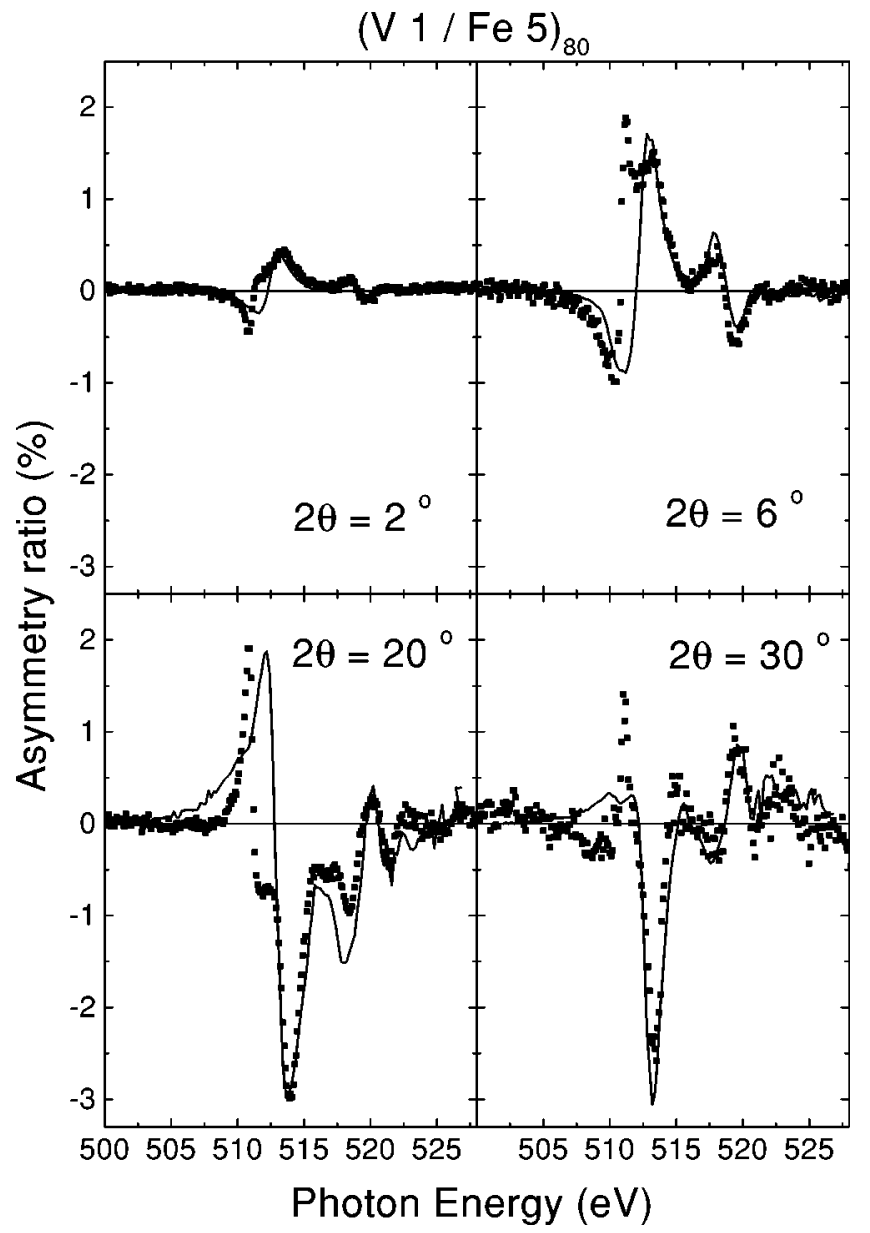

FIG. 2. Experimental (squares) and calculated (lines) asymmetry ratio curves at different scattering angles for the $\mathrm{V}_{1} / \mathrm{Fe}_{5}$ superlattice.

given spectral line shape, the dichroism in absorption is to a good approximation proportional to the magnetic moment, we can already state that the average magnetic moment of $\mathrm{V}$ in $\mathrm{V}_{n} / \mathrm{Fe}_{5}$ superlattices is reduced to $60 \%$ and then to $40 \%$ when $n$ goes from 1 to 2 , and then to 5 . In this fitting procedure we also define the sign of the dichroism in absorption necessary to reproduce the reflectivity data. This allows us to confirm the antiferromagnetic coupling between Fe and V for the three samples.

For a more quantitative analysis, we have applied sum rules to the $\mathrm{V} 2 p$ absorption spectra, once the dichroism has been scaled by the appropriate $R_{\mathrm{V}}$ factor derived from reflectivity measurements. These sum rules suffer from some intrinsic limitations, already extensively discussed when first formulated. ${ }^{20}$ Nevertheless, we believe that useful information can be extracted via this procedure.

In the following, we detail our analysis only for $1 \mathrm{ML} \mathrm{V}$, the corresponding values for $2 \mathrm{ML}$ and $5 \mathrm{ML} \mathrm{V}$ being simply scaled by $60 \%$ and $40 \%$, respectively. The integral value of the unpolarized absorption $\mu_{\text {tot }}=\mu^{+}+\mu^{-}+\mu^{0}$ is obtained by subtracting a two step function from the magnetizationaveraged V $2 p$ absorption spectra (multiplied by three). Note that we assume no linear dichroism. The two steps are separated by $7.6 \mathrm{eV}$ (spin-orbit splitting of the $\mathrm{V} 2 p$ hole), the second being half the amplitude of the first. The integrals of the dichroism over the $2 p_{3 / 2}$ and $2 p_{1 / 2}$ edges are defined as

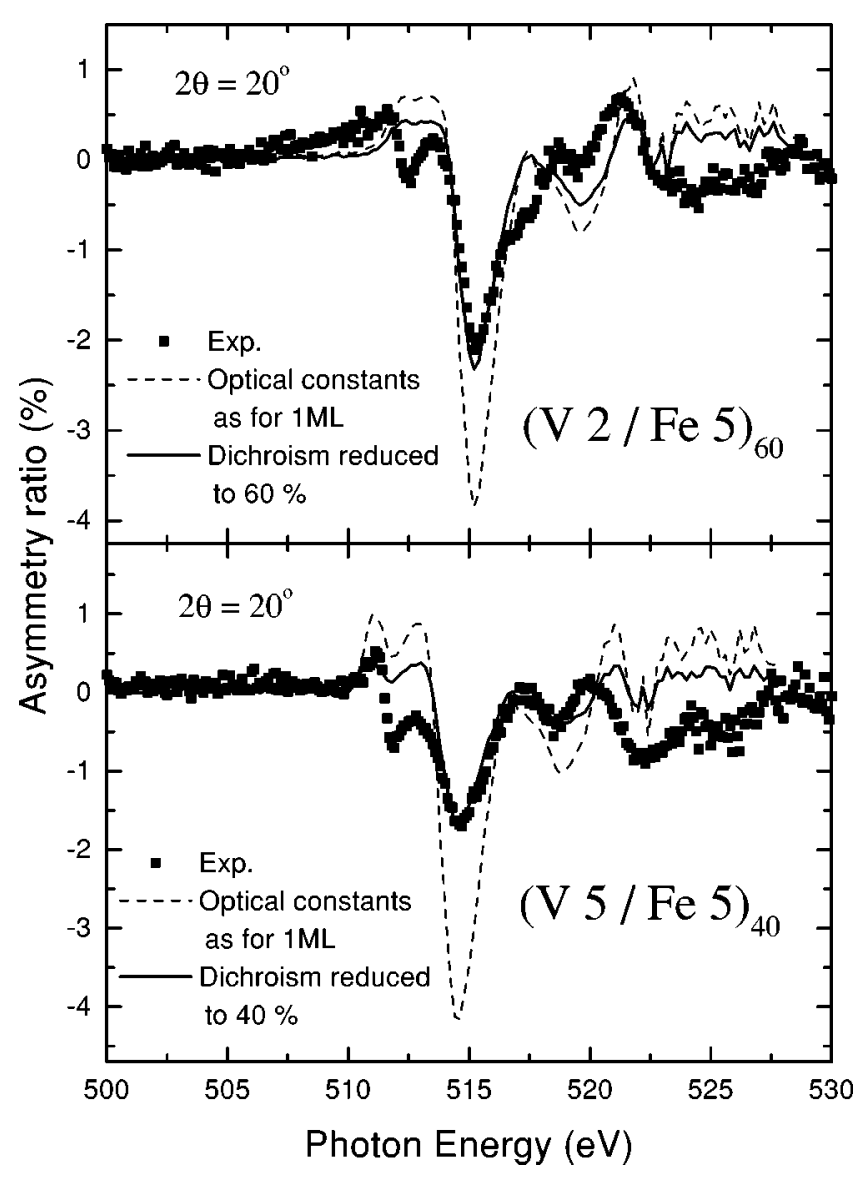

FIG. 3. Asymmetry ratio at $2 \theta=20^{\circ}$ for the $\mathrm{V}_{n} / \mathrm{Fe}_{5}(n=2$ and $n=5)$ superlattices. Squares: experimental curves; dashed lines: calculations using the same optical constants as for $n=1$; full lines: optimized calculations (see text).

$A_{3}$ and $A_{2}$, and $A$ is their sum. The first sum rule ${ }^{11}$ gives us the component along the quantization axis of the $3 d$ orbital momentum per hole: $\left\langle l_{z}\right\rangle_{\mathrm{V}} / n_{d}=2 A / \mu_{\text {tot }}=-0.013 \mu_{B}$, where $n_{d}$ is the number of $3 d$ holes in the ground state. Assuming that we can properly separate $A$ into its $A_{3}$ and $A_{2}$ components (i.e., that we can completely separate the contributions to the dichroism coming from opposite spin-orbit couplings of the core hole), the second sum rule ${ }^{12}$ gives us: $\left\langle s_{z}\right\rangle_{\mathrm{V}} / n_{d}=(3 / 2)\left(A_{3}-2 A_{2}\right) / \mu_{\text {tot }}=0.062 \mu_{B}$. In this step, we have neglected the magnetic dipolar contribution to dichroism, an assumption that is justified in cubic symmetry, ${ }^{21}$ but questionable for low dimensional systems (although it is implicit when we neglect linear dichroism). Going one step further, we can assume six holes in the vanadium $d$ band, and obtain $\left\langle l_{z}\right\rangle_{\mathrm{V}}=-0.08 \mu_{B}$ and $\left\langle s_{z}\right\rangle=0.37 \mu_{B}$, which gives a total magnetic moment per vanadium atom of $\left\langle\mu_{z}\right\rangle_{\mathrm{V}}^{1} \mathrm{ML}$ $=0.66 \mu_{B}$ for the $\left(\mathrm{V}_{1} / \mathrm{Fe}_{5}\right)_{80}$ superlattice. Even if we leave aside possible errors due to the neglect of the dipolar magnetic moment and overlap between spin-orbit split contributions, we estimate the error to be $\approx 30 \%$. Scaling according to the appropriate $R_{\mathrm{V}}$ values, we finally obtain the magnetic moments reported in the last column of Table I.

An antiparallel coupling between $\mathrm{V}$ and $\mathrm{Fe}$ moments when they are in contact is usually reported in the literature. This is probably the only point of general agreement. Finazzi et $a l .{ }^{22}$ investigated thin vanadium layers deposited on an $\mathrm{Fe}$ 
(100) single crystal using absorption spectroscopy in the TEY mode. They found a systematic reduction of the dichroism with the layer thickness, in a way consistent with the assumption that only the first vanadium layer in contact with iron carries a magnetic moment (a quantitative estimate was not given). Working on the same V/Fe (100) system using spin-polarized Auger spectroscopy, Fuchs et al. ${ }^{8}$ also observed an induced magnetic moment of about $0.3 \mu_{B}$ on the first V layer, antiparallel to Fe. On the other hand, their thickness dependent data suggest a change in sign for subsequent layers (e.g., $-0.3 \mu_{B}$ for the fourth layer), a result that has not been confirmed by other experiments.

The main qualitative and quantitative results of our XRMS experiment on $\left(\mathrm{V}_{n} / \mathrm{Fe}_{5}\right)_{m}(001)$ superlattices can be summarized as follows: (a) The magnetic moment of iron does not change with respect to its bulk value, regardless of the $\mathrm{V}$ thickness, at least to within the sensitivity of the method; (b) the coupling between Fe layers is ferromagnetic for $\mathrm{V}$ spacers up to $5 \mathrm{ML}$; (c) for all the samples under study, the V $3 d$ band carries a magnetic moment and its coupling to $\mathrm{Fe}$ is always antiferromagnetic; (d) the orbital and spin magnetic moments of the $\mathrm{V} d$ states are antiparallel, and their ratio (independent of the number of holes) is about -0.22 ; (e) for the $1 \mathrm{ML} \mathrm{V}$ sample, the orbital moment is of the order of $-0.013 \mu_{B}$ per $d$ hole, i.e., $-0.08 \mu_{B}$ assuming six $d$ holes per atom; (f) with the additional assumptions previously discussed, we obtain a V spin moment per $3 d$ hole of $0.062 \mu_{B}$, giving a total spin moment of $0.37 \mu_{B}$ and a total magnetic moment of $0.66 \mu_{B}$ per vanadium atom in the $1 \mathrm{ML} V$ sample; (g) with respect to the values for the $1 \mathrm{ML} \mathrm{V}$ sample, the magnetic moment per vanadium atom reduces to $60 \%$ and $40 \%$ in the case of the $2 \mathrm{ML}$ and $5 \mathrm{ML}$ samples.
Since we are dealing with superlattices in this paper, the most interesting comparison is with the coupled work of Harp et al. ${ }^{9}$ and Schwickert et al. ${ }^{10}$ The latter also reports model calculations comparing "perfect'" and "diffused" $\mathrm{V} / \mathrm{Fe}$ interfaces. The apparent agreement with the predictions of Schwickert et al. ${ }^{10}$ for the diffused interface case (their Table II, last column) has to be taken with caution in view of the large error bars we attribute to our measurement of the $\mathrm{V}$ magnetic moments. Even so, our estimated values fit their calculations better than their own experiments (see their Fig. 9), suggesting that interdiffusion is a critical parameter when modeling the magnetic properties of multilayers; after all, most of the sample is in fact interface.

Schwickert et al. ${ }^{10}$ also reported a consistent enhancement (about $0.5 \mu_{B}$ ) of the average Fe moment for $1 \mathrm{ML}$ vanadium, a result that neither our data nor their calculation support. Other very recent first-principles calculations by Niklasson et al. ${ }^{23}$ are in excellent agreement with our findings both for $\mathrm{V}$ and Fe.

In conclusion, we have shown, subject to the usual limitations, that sum rules developed for x-ray absorption may also be applied to resonant scattering data to extract quantitative information. In $\left(\mathrm{V}_{n} / \mathrm{Fe}_{5}\right)_{m}(001)$ superlattices we obtain a magnetic moment of about $0.66 \mu_{B}$ for $n=1$. This value diminishes as the $\mathrm{V}$ thickness increases. Because XRMS has a large depth of field and provides spectroscopic as well as structural information it is clearly a particularly relevent technique for studying magnetic multilayer devices.

The assistance of the ALS staff is gratefully acknowledged.

\footnotetext{
*Also at LURE.

†Present address: ABB Corporate Research, S-721 78 Västerås, Sweden.

${ }^{1}$ See, for instance, F. J. Himpsel et al., Adv. Phys. 47, 511 (1998).

${ }^{2}$ P. Bruno and C. Chappert, Phys. Rev. Lett. 67, 1602 (1991); 67, 2592 (1991).

${ }^{3}$ S. Y. Hsu et al., Phys. Rev. Lett. 78, 2652 (1997).

${ }^{4}$ G. Prinz, Phys. Today 48 (4), 58 (1995).

${ }^{5}$ P. Granberg et al., Phys. Rev. B 54, 1199 (1996).

${ }^{6}$ P. Isberg et al., Vacuum 48, 483 (1997).

${ }^{7}$ P. Isberg et al., Phys. Rev. B 57, 3531 (1998).

${ }^{8}$ P. Fuchs et al., Phys. Rev. B 53, 9123 (1996).

${ }^{9}$ G. R. Harp et al., Phys. Rev. B 51, 3293 (1995).

${ }^{10}$ M. M. Schwickert et al., Phys. Rev. B 57, 13681 (1998).

${ }^{11}$ B. T. Thole et al., Phys. Rev. Lett. 68, 1943 (1992).

${ }^{12}$ P. Carra et al., Phys. Rev. Lett. 70, 694 (1993).

${ }^{13}$ J. Vogel and M. Sacchi, J. Electron Spectrosc. Relat. Phenom. 67,
}

181 (1994); Phys. Rev. B 49, 3230 (1994); V. Chakarian et al., ibid. 57, 5312 (1998); R. Nakajima et al., ibid. 59, 6421 (1999).

${ }^{14}$ J.-M. Tonnerre et al., Phys. Rev. Lett. 75, 740 (1995).

${ }^{15}$ M. Sacchi et al., Phys. Rev. B 57, 108 (1998); Phys. Rev. Lett. 81, 1521 (1998)

${ }^{16}$ L. Sève et al., Phys. Rev. B 60, 9662 (1999).

${ }^{17}$ M. Sacchi and A. Mirone, Phys. Rev. B 57, 8408 (1998).

${ }^{18}$ P. Isberg, Ph.D. thesis, Uppsala University, 1997.

${ }^{19}$ J. H. Underwood and E. M. Gullikson, J. Electron Spectrosc. Relat. Phenom. 92, 265 (1998).

${ }^{20}$ See, for instance, R. Wu et al., Phys. Rev. Lett. 71, 3581 (1993); R. Wu and A. J. Freeman, ibid. 73, 1994 (1994); C. T. Chen et al., ibid. 75, 152 (1995).

${ }^{21}$ P. Carra et al., Physica B 192, 182 (1993).

${ }^{22}$ M. Finazzi et al., J. Magn. Magn. Mater. 165, 78 (1997).

${ }^{23}$ A. M. N. Niklasson et al., Phys. Rev. B 59, 6373 (1999). 